\title{
Landmark use by squirrel monkeys (Saimiri sciureus)
}

\author{
JENNIFER E. SUTTON, ANNEKE OLTHOF, and WILLIAM A. ROBERTS \\ University of Western Ontario, London, Ontario, Canada
}

\begin{abstract}
Two squirrel monkeys searched for a reward buried in 1 of 144 holes that formed a $12 \times 12$ grid (48 $\times$ $50 \mathrm{~cm}$ ). An array of vertical, colored landmarks was placed on the grid, and their locations on the grid were changed from trial to trial. During training trials, the mealworm reward was placed either in the center of a square array of landmarks (Experiments 1 and 3) or midway between two landmarks (Experiment 2). On nonrewarded test trials, the monkeys searched among landmarks placed in the same arrays as those used in training and among landmarks placed in an expanded array (Experiments 1 and 2) or in an array intermediate between the two arrays used in training (Experiment 3). Distributions of searches on test trials indicated that the monkeys searched mostly within the configuration of the landmarks but that they had not coded the location of the reward as being either in the middle of the landmarks or at a fixed distance and direction from an individual landmark.
\end{abstract}

A crucial form of information for people and animals is position in space. Appropriate orientation and navigation are essential for successful foraging and predator evasion, among other things. A number of studies have investigated how a variety of species use visual information to encode spatial locations (see Roberts, 1998, for a review). For example, Cartwright and Collett (1983) trained bees to find a cup of sucrose that was placed equidistant from three black cylindrical landmarks. The landmarks and the sucrose were moved to a different location in the testing room for each trial, so that the landmarks were the only consistent cue for the location of the sucrose. After the bees reliably found the sucrose, the landmark array was altered on test trials by shortening or lengthening the distance between the cylinders. On these test trials, the sucrose was not present, and the bees' flight path was recorded. When the array was expanded, the bees searched farther away from the landmarks than they did in training, and when it was contracted, the bees searched closer to the landmarks.

Cartwright and Collett (1983) suggested that the bees used a simple method to estimate the location of the sucrose on test trials: They searched in a location from which the landmarks could be visually perceived as being in the same compass directions as they were from the goal location in training. The bees seemed to have formed a "snapshot" of the training array as viewed from the goal location and then to have referred to that snapshot memory when searching on test trials. Consequently, when the array was expanded, the bees searched farther away so that the test array would appear similar to the

This research was supported by a research grant from the Natural Sciences and Engineering Research Council of Canada. Correspondence concerning this article should be addressed to W. A. Roberts, Department of Psychology, University of Western Ontario, London, Ontario N6A 5C2, Canada (e-mail: roberts@julian.uwo.ca). original training configuration, and they searched close when the array was contracted, to make the landmark appear similar to the training array.

This method of training with a moving array of land marks followed by tests with an altered array has beel used to test other species as well and has revealed differ ent strategies for locating a goal by using such visua cues. In one experiment, Collett, Cartwright, and Smitl (1986) trained gerbils to find a sunflower seed buried it bedding at a location that was fixed relative to two land marks. When the distance between the landmarks wa lengthened on test trials with no sunflower seed, the ger bils tended to search at two locations, rather than at one The locations of their searches indicated that the gerbil had computed the distance and direction of the goal fron each landmark independently. Gerbils did not encode thr goal location as a point equidistant from both landmark or as a two-dimensional snapshot of the array.

Using a similar task, Spetch et al. (1997) found tha pigeons encoded the location of a buried seed in a simi lar fashion. In one experiment, pigeons were trained ts find buried maple peas located in the center of four land marks that formed a square. On test trials, the array wa: expanded to form a larger square. The pigeons used : strategy similar to that used by the gerbils in Collet et al.'s (1986) experiment. Searches were concentrated a points that preserved the direction and distance from in dividual landmarks to the goal independent of the others Although pigeons could have learned that the goal was a a point equidistant from each of the four landmarks, they searched for the goal with respect to each landmark in dividually. When Spetch et al. (1997) tested humans or a similar task, they continued to search in the center o the array on test trials with expanded arrays. Unlike ger bils and pigeons, people coded the goal location as : point equidistant from the landmarks and continued tc search there when the array was expanded. 
The present experiments expanded the variety of species tested with this paradigm to include a nonhuman primate, Saimiri sciureus, or the squirrel monkey. Little is known about the use of landmarks by nonhuman primates, and the sharp difference in strategies employed by humans and other animals on landmark tests invites a study of the strategy used by a species phylogenetically closer to humans than were the species tested thus far. Saimiri are New World monkeys and are classified as omnivorous frugivores that consume fruit, leaves, insects, and nuts. Saimiri troops have been observed to spend up to $95 \%$ of their waking hours traveling and rapidly foraging in their natural Central and South American rain forest habitat (Baldwin \& Baldwin, 1972). Laboratory spatial memory tasks with these primates have revealed a tendency to sample all of the available alternatives, instead of precisely returning to a consistently baited location (Roberts, Mitchell, \& Phelps, 1993). Nonetheless, given the importance of spatial information for foraging and traveling in a complex natural environment, the strategies used by these monkeys in a landmark foraging task are of interest. It may be that their rapid, constant foraging behavior in nature necessitates only the most general reliance on spatial cues. In the present experiments, we investigated the search patterns of two squirrel monkeys, using landmarks as visual cues for the location of a goal. We particularly wanted to find out whether the search strategy employed by the monkeys would resemble that used by humans or that used by pigeons and gerbils.

\section{EXPERIMENT 1}

In Experiment 1, we investigated the ability of 2 squirrel monkeys to locate a hidden goal by using a configuration of landmarks similar to that used by Spetch et al. (1997) with pigeons and humans. The monkeys were trained to locate a mealworm buried in oatmeal at the center of an array of four cylindrical landmarks. The landmark array was in a different location on a foraging board $(48 \times 50 \mathrm{~cm})$ on each trial, with the mealworm always located in the center of the array. Nonrewarded test trials were carried out, in which the array was either expanded diagonally to three times its size in training or was left at the same size as in training. Monkeys searched for $1 \mathrm{~min}$ on test trials, and the location of the searches was recorded on each trial. The primary question of interest was where the monkeys would focus their searches on test trials with expanded arrays.

\section{Method}

Subjects. Two 11-year-old male squirrel monkeys, named Jake and Elwood, participated in this research. Elwood previously had participated in research on face memory (Phelps \& Roberts, 1994), and both monkeys had participated in a study of foraging in a simulated tree environment (Roberts et al., 1993) and in a study of ordinality and summation of number symbols (Olthof, Iden, \& Roberts, 1997). The monkeys were housed in individual cages at the University of Western Ontario and were allowed to interact with one another in a large play cage with swings and toys for $3-4 \mathrm{~h}$ six times per week. They were kept on a 13:11-h light:dark schedule, with light onset at 7 a.m. and offset at 8 p.m. each day. Their diet consisted of Monkey Chow (Ralston-Purina, St. Louis, MO), supplemented with various fruits, vegetables, and vitamins. The monkeys were not food deprived during any of these experiments. They were tested $1 \mathrm{~h}$ prior to their daily feeding 5-6 times per week. All care and feeding of the monkeys was in strict accordance with Canadian Council on Animal Care guidelines.

Apparatus. The monkeys were trained and tested in a modified test chamber, which measured $49.5 \mathrm{~cm}$ wide $\times 51.0 \mathrm{~cm}$ long $\times$ $52.0 \mathrm{~cm}$ high and was located on a table in a testing room. One side of the chamber and the ceiling were made of clear acrylic. The monkeys entered the apparatus via a vertically sliding door located on one wall. A white acrylic foraging board, $48 \mathrm{~cm}$ wide $\times 50 \mathrm{~cm}$ long $\times 2 \mathrm{~cm}$ thick served as the entire floor of the chamber, so that the monkeys moved around directly on it during a trial. The board had 144 holes drilled into it in a $12 \times 12$ grid. Each hole was $2 \mathrm{~cm}$ in diameter and $1.5 \mathrm{~cm}$ deep. All the holes in the board were filled with dry Quaker brand oatmeal. Four cylindrical wooden lengths of doweling, $13.5 \mathrm{~cm}$ high and $1.8 \mathrm{~cm}$ in diameter, served as landmarks and were covered in blue construction paper. These landmarks could be inserted into the holes on the board and moved from trial to trial. All the trials were recorded through the clear acrylic side wall, using a video camera.

Procedure. Each monkey was first trained to remove oatmeal from a hole $1.5 \mathrm{~cm}$ deep, drilled in a small block of wood, to find a buried mealworm. Initially, the mealworm was covered by only a small amount of oatmeal but was gradually covered more on successive trials until the monkeys were consistently removing all the oatmeal and obtaining the mealworm when it was completely covered. This initial training lasted approximately 1 week.

The monkeys then were trained to find a mealworm buried in one of the holes on the foraging board in the test chamber. The hole with the buried mealworm was always located in the center of an array of four landmarks. The array formed a square so that the landmarks were one hole apart on each side, as is illustrated in the top panel of Figure 1. The landmarks were placed in a different location on the foraging board on each trial, and each session consisted of five trials. The mealworm was only partially covered by oatmeal on the first few trials of Sessions 1-7 and was completely covered and buried in the bottom of the hole on every trial in subsequent training sessions. On a trial, the monkey entered the box, searched in the holes on the board for a maximum time of $1 \mathrm{~min}$, and then exited the box and was given a peanut. A search was defined as the removal of oatmeal from a hole with either the monkey's left or its right hand. Multiple searches to the same hole were counted only if the monkey searched in another location between repeat searches (i.e., repeated digging in a single hole was counted as one search). The location of each search was recorded. If the monkey did not find the mealworm within $1 \mathrm{~min}$ on the first attempt, the mealworm was uncovered, and the monkey was allowed to reenter the apparatus and obtain it. Training lasted for 28 sessions, with 1 session per day.

Test sessions consisted of five trials per session composed of a random order of two test trials and three training trials, with the restriction that the first trial of a session was always a training trial. No mealworm was present on test trials. On one test trial, the array was in the same configuration as that in training (training configuration) and was identical to a training trial with one hole between each landmark. On the other test trial, the array was expanded so that it formed a square with three holes between each landmark (expanded configuration), as is shown in the bottom panel of Figure 1. The monkeys were tested on each test trial type 20 times. The location of the test configuration on the board varied across trials, and the order of test trial type within a session varied randomly across sessions. The monkeys were given $1 \mathrm{~min}$ to search on each 


\section{Training Configuration}

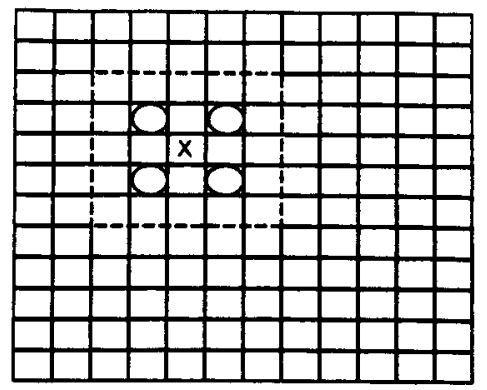

\section{Expanded Configuration}

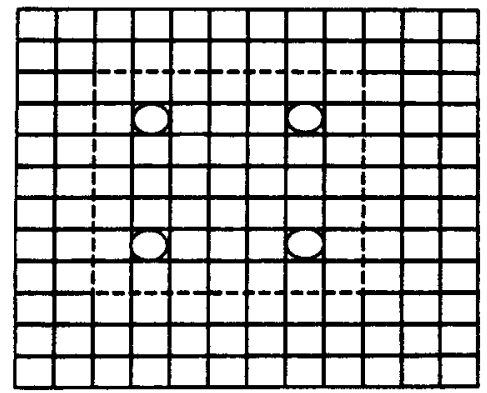

Figure 1. Examples of the training configuration (top panel) and expanded configuration (bottom panel) used in Experiment 1 . The foraging board was a $12 \times 12$ grid of holes filled with oatmeal. Circles represent the placement of the four cylindrical landmarks, which could be inserted into holes on the board. The " $x$ " denotes the location of the reward on training trials. The entire configuration was in a different location on the board on each trial. Unbaited test trials were conducted with the training and expanded configurations. The dashed line indicates the landmark boundary within which response patterns were examined.

test trial. The trials were recorded with a video camera, and the location of each search was noted.

\section{Results and Discussion}

In the last five sessions of training, Jake found the completely covered mealworm within $1 \mathrm{~min}$ on $100 \%$ of the trials, with a mean of 8.0 searches $(S D=2.17)$ to find the goal. Elwood found the completely covered mealworm within $1 \mathrm{~min}$ on $96 \%$ of the trials in the last five sessions of training, with a mean of 5.20 searches $(S D=$ 1.48 ) on trials in which the goal was found. The observation that the monkeys took 5-8 searches to find the mealworm was not based entirely on searches within the landmark array. Both monkeys tended to dig routinely in several holes on the way to the landmark array and thus to increase the overall number of searches that were required to find the reward.

The monkeys' search locations on test trials were analyzed as being either within a one-hole perimeter of the landmark array or beyond this area on the rest of the foraging board, as is illustrated in Figure 1. The number of searches per hole within an area was obtained by dividing the total number of holes searched in that area by the total number of holes available in that area. Averaged over all the "searchable" (i.e., not occupied by a landmark) holes, Jake made a mean of .77 searches per hole $(S D=.23)$ within the landmark boundary area and a mean of .06 searches per hole $(S D=.03)$ within the area outside the landmark boundary on control test trials. On expanded test trials, Jake made a mean of .41 searches per hole $(S D=.09)$ within the landmark boundary area and a mean of .07 searches per hole $(S D=.04)$ outside the landmark boundary area. For all statistical tests, alpha was set at .05 . According to $t$ tests, Jake searched significantly more often within the array boundary than outside it on both types of trial [control, $t(19)=12.76$; expanded, $t(19)=13.77]$.

On control test trials, Elwood made a mean of .67 searches per hole $(S D=.19)$ within the landmark boundary area and a mean of .04 searches per hole $(S D=.03)$ outside the landmark boundary area. On expanded test trials, Elwood made a mean of .40 searches per hole $(S D=.11)$ within the landmark boundary area and a mean of .05 searches per hole $(S D=.03)$ outside the landmark boundary area. On both types of trial, Elwood searched significantly more within the array boundary than outside it [control, $t(19)=13.21$; expanded, $t(19)=$ 12.09]. A tendency for both monkeys to search in holes outside the array area boundary was initially noted as they moved toward the landmark area, with some searches outside the area occurring during the rest of the trial in no discernable pattern on the board. Also, neither monkey exhibited a consistent behavioral sequence of visits to the holes within either array.

In addition, it was determined whether the monkeys searched within the boundary around the array more than would be expected if they were searching randomly (i.e., whether they searched there a higher proportion of times than would be expected by chance alone). Chance was calculated by dividing the number of searchable holes within the boundary area by 140 (the total number of holes on the board minus the four holes with landmarks). The proportion of searches within the boundary around the array if the monkeys were searching randomly would be .15 on control test trials and .32 on expanded test trials. Jake's mean proportion of searches to the area within the boundary was $.70(S D=.15)$ on control tests and $.74(S D=.14)$ on expanded tests; both means were significantly greater than chance [control, $t(19)=16.23$; expanded, $t(19)=13.59$ ]. Elwood's mean proportion of searches to the area within the boundary was $.74(S D=.18)$ on control tests and $.79(S D=.13)$ on expanded tests, and both means were significantly above chance [control, $t(19)=14.19$; expanded, $t(19)=17.05$ ]. The results of these tests suggest that the monkeys' search patterns were strongly influenced by the position of the landmarks on the foraging board.

Searches within the boundary were further examined by plotting the total number of searches for each hole within the boundary area and are depicted in three- 

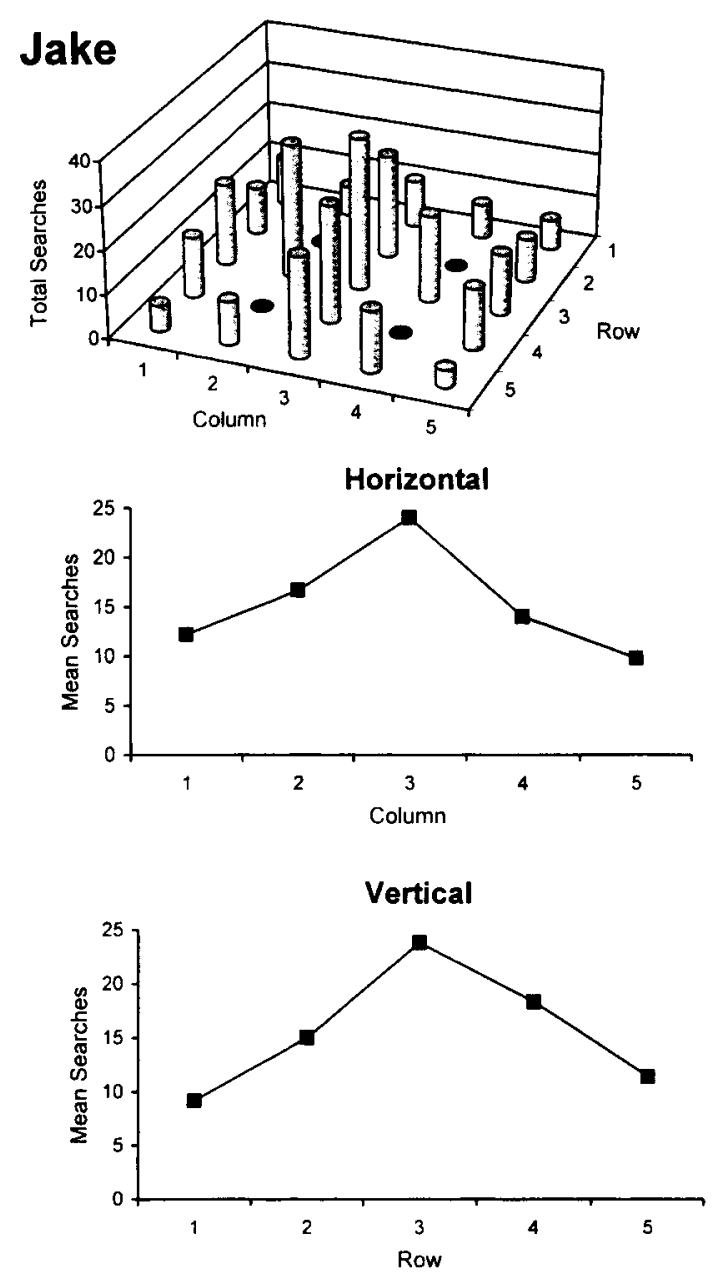
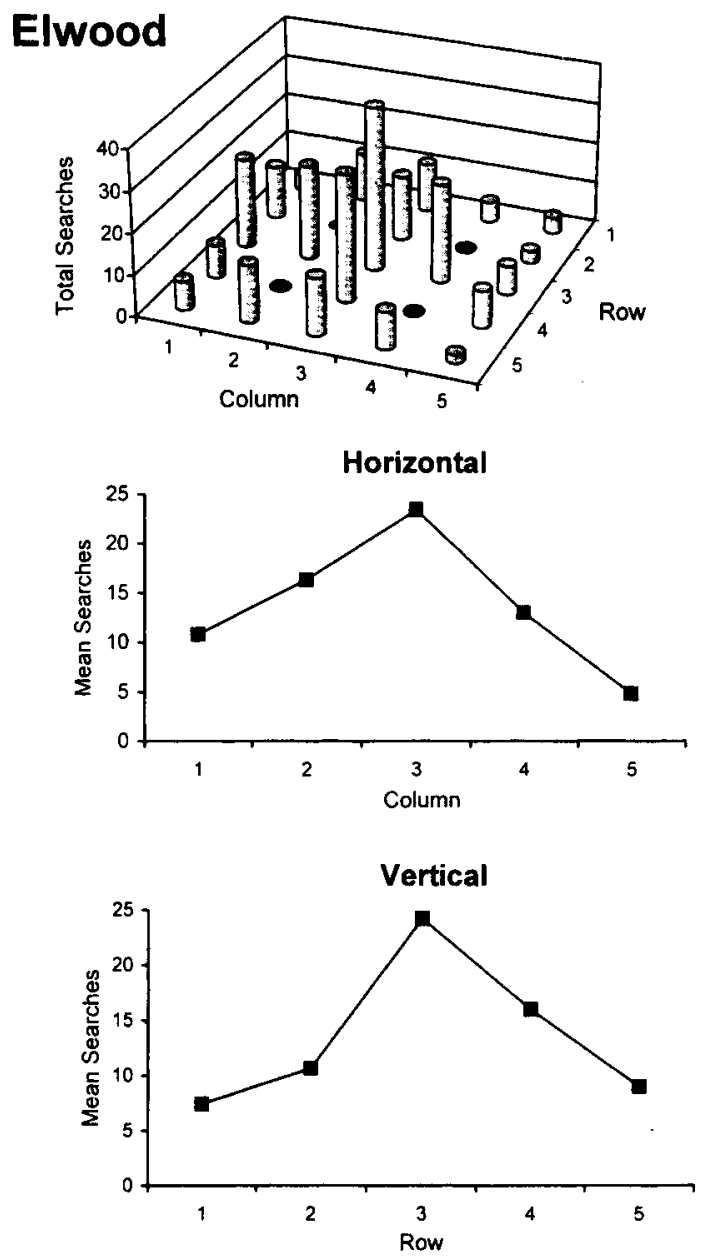

Figure 2. Total searches to each hole within the control array boundary and mean searches in columns (horizontal) and rows (vertical) for Jake and Elwood on control array test trials in Experiment 1. Landmark locations are denoted with filled black circles in the three-dimensional graphs.

dimensional format in Figure 2. As was expected, both monkeys directed most of their searches to the center hole on control array tests, with Elwood's search pattern producing a clearer peak than Jake's for the center hole of the array. Also shown are the mean number of searches for the horizontal (columns) and vertical (rows) dimensions within the array area in two-dimensional format. Analyses of variance (ANOVAs) revealed significant differences in mean searches for both monkeys across the horizontal dimension [Jake, $F(4,76)=7.55$; Elwood, $F(4,76)=15.01]$ and along the vertical dimension [Jake, $F(4,76)=9.24$; Elwood, $F(4,76)=13.12$ ]. Post hoc Tukey HSD tests were conducted on columns and rows to further determine where the monkeys focused their searches. Along the horizontal dimension, Jake searched significantly more in the center column (3) than in Columns 1, 4, or 5. Elwood searched significantly more in the center column than in Columns $1,2,4$, or 5 . He also searched more in the columns that contained landmarks (Columns 2 and 4) than in Column 5.
Along the vertical dimension, both monkeys searched in the center row (3) significantly more than in Row 1, 2, or 5. In addition, Elwood searched more in the center row than in Row 4 and more in Row 4 than in Row 1. These post hoc tests confirm that the monkeys generally focused their searches in the center row and column of the control test array, although Jake did not always search significantly more in the center row or column than in a neighboring row or column. Elwood's tendency to search toward the bottom of the array (Row 4) rather than the top (Row 1) is not surprising, since the monkeys usually approached the array from that direction after entering the chamber.

Figure 3 shows the search distribution on the expanded array tests for each monkey. An inspection of the three-dimensional response distributions reveals that both monkeys' searching was less localized on expanded array tests than on the control array tests. The twodimensional graphs show a different pattern of searches over columns and rows than was found on control array 

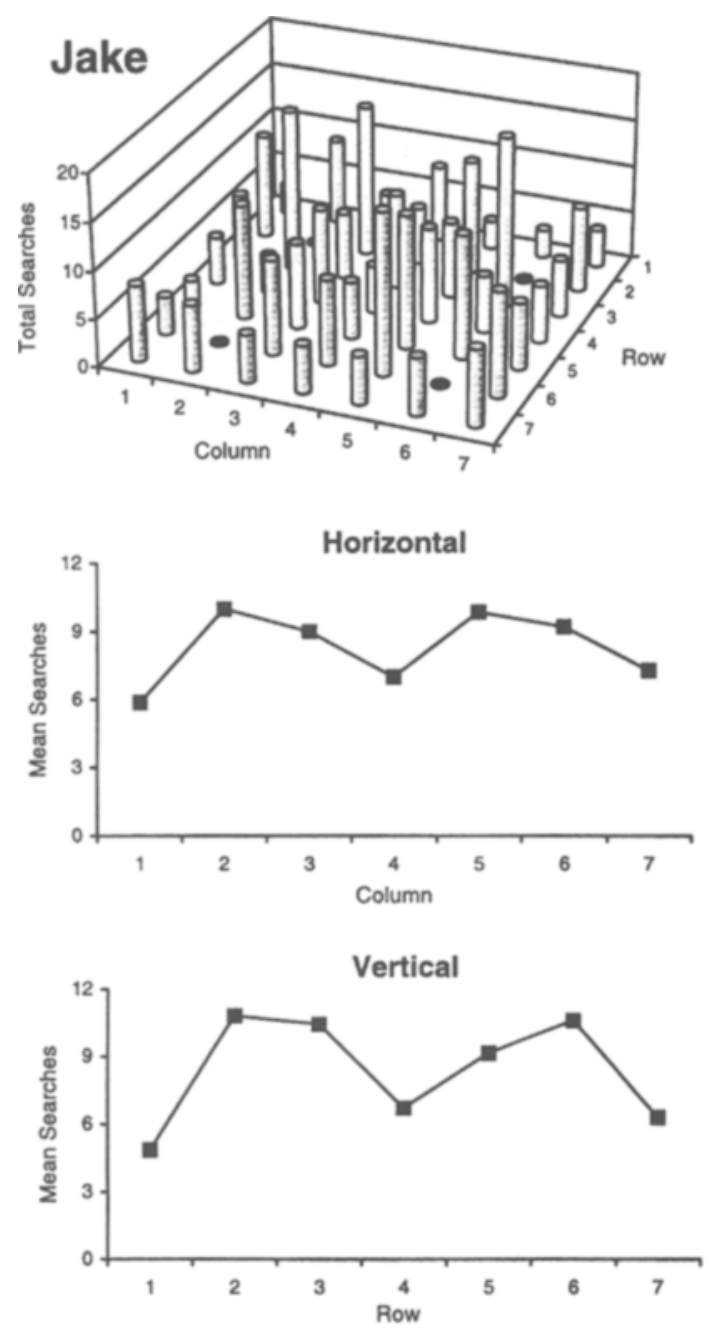
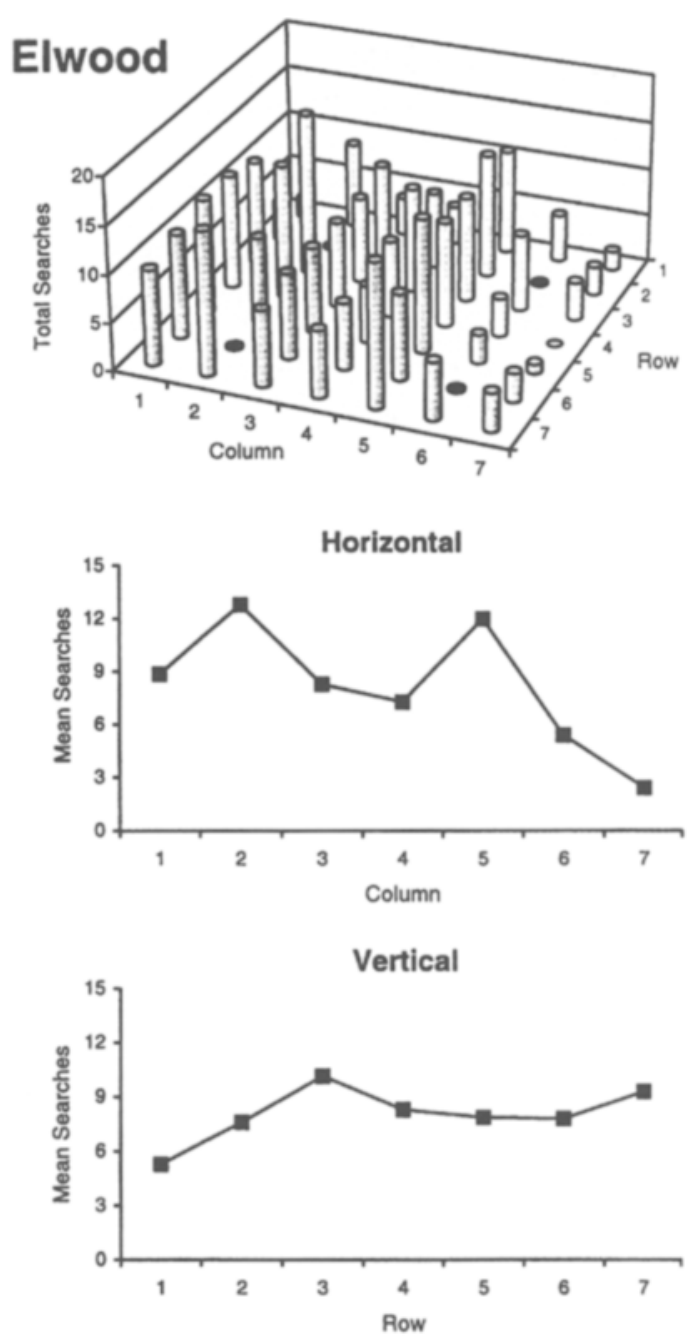

Figure 3. Total searches to each hole within the array boundary and mean searches in columns (horizontal) and rows (vertic for Jake and Elwood on test trials with expanded arrays in Experiment 1. Filled black circles denote landmark locations.

tests. Along the horizontal dimension, Jake's searches did not differ significantly across columns, whereas Elwood's did $[F(6,114)=7.04]$. Post hoc Tukey HSD tests revealed that Elwood searched more in Column 2 (a column with landmarks) than in Columns 6 or 7 . He also searched in Column 5 (just to the left of the right landmark) significantly more often than in Columns 6 or 7 and in Column 1 more than in Column 7. Along the vertical dimension, Elwood's searches did not differ significantly over rows, whereas Jake's did $[F(6,144)=3.35]$. Post hoc Tukey HSD tests showed that Jake searched significantly more in Rows 2, 3, and 6 than in Row 1 .

The two-dimensional analyses confirm that, on expanded array tests, the monkeys tended to focus their searches in rows and columns that contained landmarks or in rows and columns adjacent to landmarks, or else they failed to significantly differentiate between columns (Jake) or rows (Elwood). The general pattern is different from the patterns observed with the control array tests in
Figure 2, where monkeys tended to search the mos the center hole. It may be that the location of the goa training was encoded as a location adjacent to one more landmarks on the inside of the array, a strategy $t$ carried over but became less focused when applied to expanded test arrays. The three-dimensional graph Elwood also shows that he tended to search more on left side of the expanded array. The reason for this $t$ is not clear; consistent avoidance of one area of chamber is an unlikely explanation, since the array 1 in a different location on the foraging board on e; trial.

It is clear that neither monkey searched more in center hole of the expanded array than in other holes. would be expected if the monkeys used the "search in middle" strategy found in humans (Spetch et al., 199 Searches directed to the landmarks were consistent w results from studies with pigeons (Spetch et al., 19! and gerbils (Collett et al., 1986), but the overall lack 
focus in the monkeys' responses was not. The monkeys seemed to be using the landmarks as cues for the location of the mealworm, but in a fairly general, rather than localized, way.

\section{EXPERIMENT 2}

Experiment 1 revealed that, although the monkeys' searches were influenced by the landmark array, in that they searched near the configuration more than in other places on the board, there were no clear, highly localized peaks of responding in individual holes on expanded test trials, such as have been reported with other species. In Experiment 2, the monkeys were trained to find a mealworm buried between only two landmarks separated by a fixed distance and were subsequently tested with the landmarks moved farther apart. This configuration was similar to the configuration used by Collett et al. (1986) to test gerbils.

The change to two landmarks from four was an attempt to encourage more localized searching by the monkeys on test trials. It was reasoned that fewer landmarks might result in greater attention to the existing landmarks and, consequently, to the spatial relationship between the landmarks and the mealworm goal. Also, the configuration resulted in a straight line between the landmarks, with the mealworm being located at the midpoint. It is possible that it could be simpler for the monkeys to learn this relationship than the center of a fourlandmark square configuration. Finally, by training with three holes between the landmarks instead of one, a tendency to search close to individual landmarks on expanded test trials might not be as pronounced as it was in Experiment 1.

\section{Method}

Subjects. The same squirrel monkeys and apparatus as those used in Experiment 1 were used in Experiment 2, except that only two lengths of wooden doweling were used as landmarks, and they were covered with yellow construction paper.

Procedure. The training for Experiment 2 began immediately after the completion of Experiment 1 . The monkeys were trained to find a mealworm buried in oatmeal in a hole between two yellow landmarks. The landmarks were separated by three holes and were placed in a different randomly determined location on the board for each trial. The orientation of the landmarks was always parallel to the wall containing the door through which the monkeys entered the chamber. The landmark configuration is illustrated in Figure 4. The mealworm always was located in the hole at the midpoint of the landmark array. There were five trials per session, and one session was conducted per day. The mealworm was only partially covered by oatmeal on the first few trials of Sessions 1-6 for Jake and Sessions 1-4 for Elwood and was completely buried in the bottom of the hole on every trial in subsequent training sessions for both monkeys. The trials proceeded as in Experiment 1: The monkeys entered the test chamber, searched through the holes for $1 \mathrm{~min}$ or until the mealworm was found, and then exited the test chamber and received a peanut. If the monkey did not find the mealworm within $1 \mathrm{~min}$ on the first attempt, the mealworm was uncovered, and the monkey was allowed to reenter the apparatus and obtain it. Training continued for 25 sessions.

Test sessions consisted of five trials per session, consisting of a random order of two test trials and three training trials, with the restriction that the first trial of a session was always a training trial. No mealworm was present on test trials. On one test trial, the array was in the same configuration as that in training (training configuration) and was identical to a training trial (but unbaited), with three holes between the landmarks. On the other test trial, the array was expanded so that there were five holes between the landmarks (expanded configuration), as is shown in Figure 4. The monkeys were tested on each type of test trial 20 times and were given 1 min to search on each test trial. The trials were recorded with a video camera, and the location of each search was noted.

\section{Results and Discussion}

In the last five sessions of training, Jake found the completely covered mealworm within 1 min on $100 \%$ of the trials, with a mean of 7.6 searches $(S D=3.36)$ to find the goal. Elwood found the completely covered mealworm within $1 \mathrm{~min}$ on $95 \%$ of the trials in the last five sessions of training, with a mean of 6.2 searches $(S D=3.56)$.

As in Experiment 1, the monkeys' search locations on test trials were analyzed as being either within a one-hole perimeter of the landmark array or beyond this area on

\section{Training Configuration}

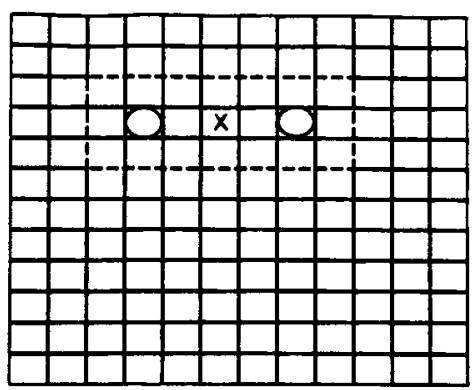

\section{Expanded Configuration}

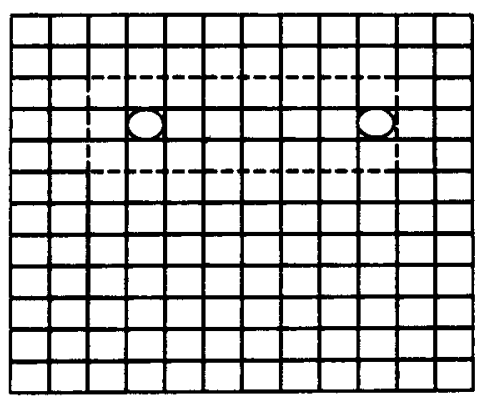

Figure 4. Examples of the training configuration and expanded configuration of landmarks used in Experiment 2. Circles show the positions of the landmarks, and the dashed line indicates the boundary within which response patterns were examined. The position of the reward on training trials only (there was no reward on test trials) is denoted by an "x." 
the rest of the foraging board, as is illustrated by the dashed lines in Figure 4. On control test trials, Jake made a mean of .77 searches per hole $(S D=.13)$ within the landmark boundary and a mean of .11 searches per hole $(S D=.04)$ on the rest of the board. On expanded test trials, he made a mean of .74 searches per hole $(S D=.15)$ within the landmark boundary and a mean of .10 searches per hole $(S D=.03)$ outside the landmark boundary. Jake made significantly more searches to holes inside the landmark boundary than to holes on the rest of the board on both types of tests [control, $t(19)=19.61$; expanded, $t(19)=17.28]$. On control tests, Elwood made a mean of .47 searches per hole $(S D=.11)$ within the landmark boundary and a mean of .06 searches per hole $(S D=.05)$ outside the landmark boundary. On expanded array tests, Elwood made a mean of .45 searches per hole $(S D=.15)$ within the landmark boundary and a mean of .05 searches per hole $(S D=.03)$ on the rest of the board. Elwood made significantly more searches to holes inside the landmark boundary than to holes outside the boundary on both types of tests [control, $t(19)=14.73$; ex- panded, $t(19)=10.95$ ]. As in Experiment 1, the monkeys did not show any consistent sequence of visits to holes within the array boundary on either the control or the expanded array, and searches outside the boundary area tended to occur as the monkeys initially moved toward the area or at random locations during the rest of the trial.

The proportion of searches within the landmark boundary expected on the basis of chance alone was determined to be .13 for the control array and .18 for the expanded array, using the same method of calculation as that used in Experiment 1. Jake directed a higher proportion of searches to the area within the landmark boundary around the array on control tests $(M=.55$, $S D=.13)$ and on expanded tests $(M=.60, S D=.10)$ than would be expected by chance [control, $t(19)=14.40$; expanded, $t(19)=17.69$ ]. Elwood also directed a higher proportion of searches to the area within the landmark boundary on control tests $(M=.60, S D=.20)$ and on expanded tests $(M=.65, S D=.21)$ than would be expected by chance [control, $t(19)=10.25$; expanded, $t(19)=$
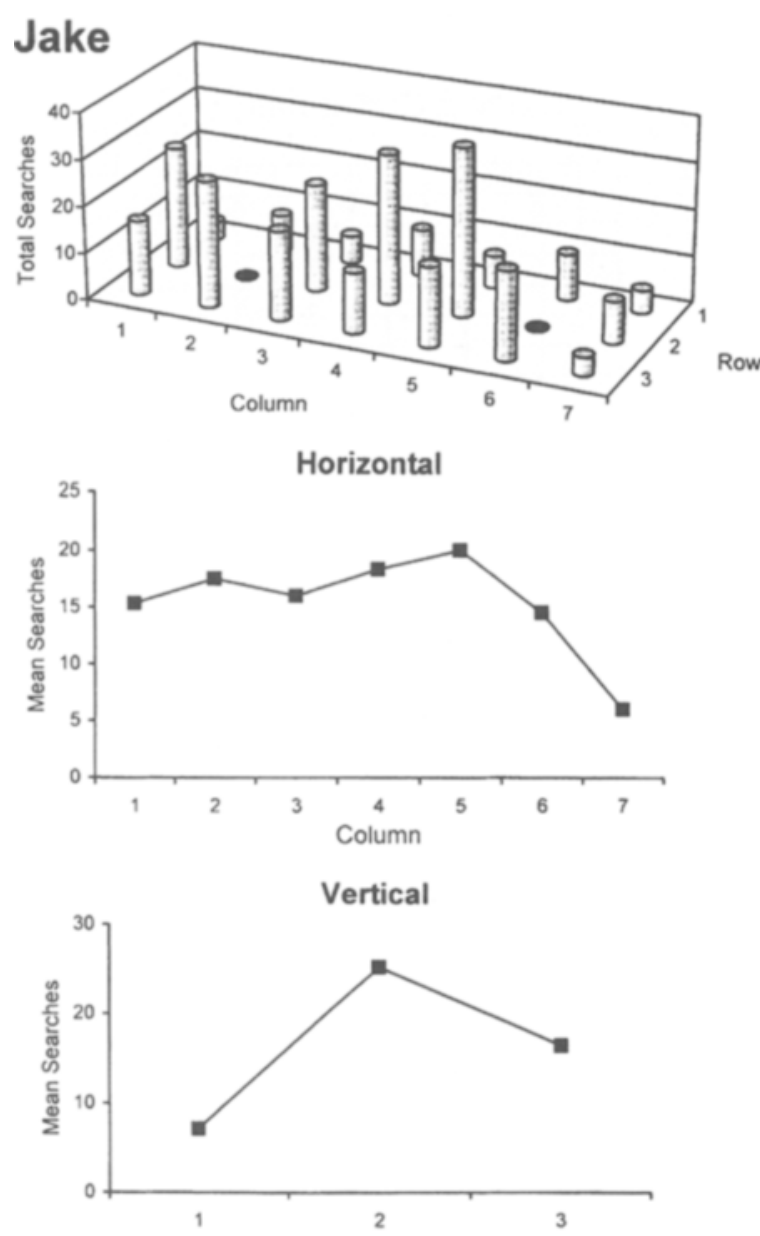
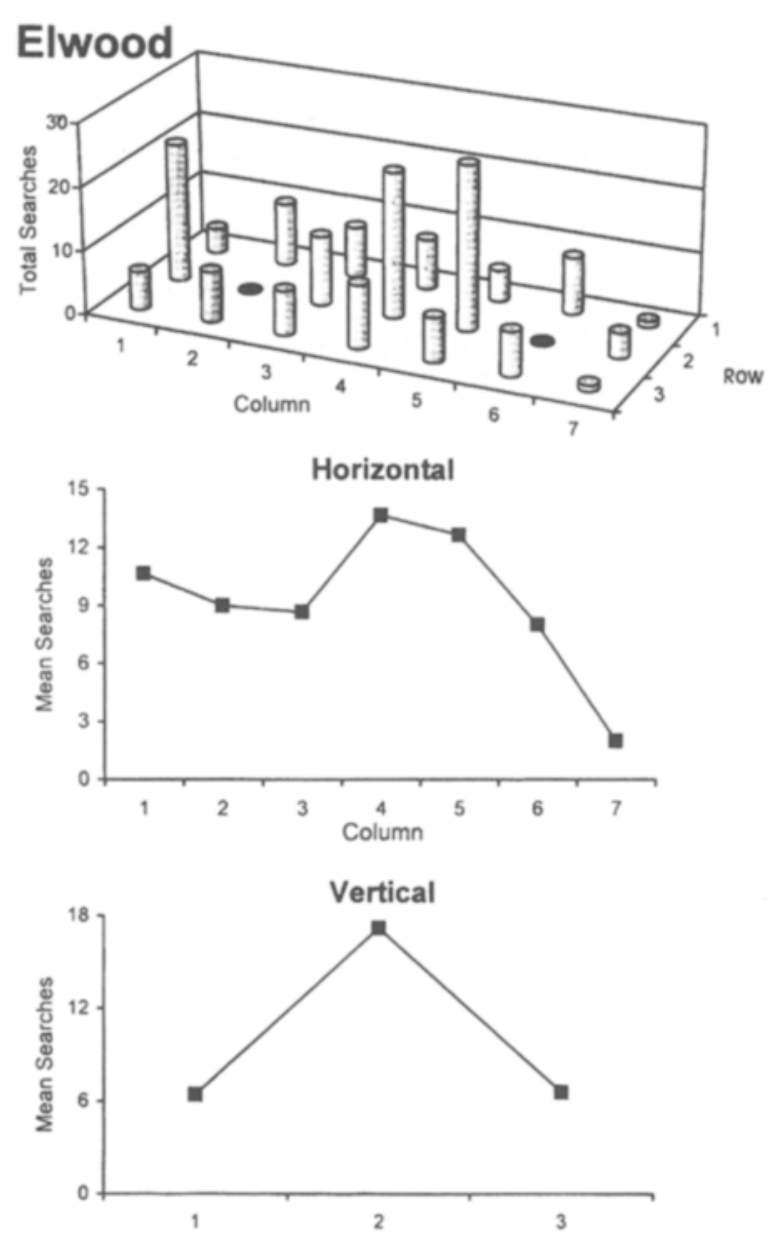

Figure 5. Total searches to each hole within the array boundary and mean searches in columns (horizontal) and rows (vertical) for Jake and Elwood on control array test trials in Experiment 2. Filled black circles denote landmark locations. 
9.67]. These results indicate that the monkeys were strongly influenced by the position of the array on both types of trials.

Search distributions for the control test trials for each monkey are shown in Figure 5 in both three-dimensional and two-dimensional formats. The three-dimensional graphs illustrate a tendency for both monkeys to search most often in the hole immediately to the left of the right landmark. ANOVAs performed on the two-dimensional data for the horizontal dimension indicated that both monkeys' search patterns differed significantly across columns [Jake, $F(6,114)=4.76$; Elwood, $F(6,114)=$ 4.82]. Post hoc Tukey HSD tests showed that significant differences between the mean number of searches in columns were mostly limited to differences between the far-right column (7) and the others. For instance, Jake searched significantly more in Columns $1,2,3,4$, and 5 than in Column 7 . Elwood searched significantly more in Columns 1, 4, and 5 than in Column 7. Further analyses were performed on the horizontal dimension data for both monkeys by performing an ANOVA on the mean searches for each hole in the middle row only. Jake's mean number of searches was significantly different over the five holes in the middle row $[F(4,76)=7.80]$, as was Elwood's $[F(4,76)=12.74]$. Tukey HSD tests revealed that Jake searched significantly more in Holes 1 , 4 , and 5 than in 7 (denoted by their respective column numbers), whereas there were no other significant differences between holes. Elwood searched significantly more in Holes 1, 4, and 5 than in Holes 3 and 7. Interestingly, these tests show that Jake's searches in holes between the landmarks in the center row did not differ significantly, whereas Elwood searched significantly more in the center hole (4) and the hole to the left of the right landmark (5) than in the hole to the right of the left landmark (3).

An ANOVA conducted on the vertical dimension showed significant differences across rows for both monkeys [Jake, $F(2,38)=4.08$; Elwood, $F(2,38)=$ 52.41]. Post hoc Tukey HSD tests confirmed that both monkeys clearly focused their searches in the center row. Jake searched in the center row (2) significantly more often than in Rows 1 and 3 and more in Row 3 than in Row 1. Elwood searched significantly more in Row 2 than in Rows 3 and 1. The pattern for both monkeys indicates a focus on the row containing the landmarks. Jake's tendency to search more toward the bottom of the array than toward the top is not surprising, given that the monkeys generally approached the array from this direction.

Although their search patterns did not show a clear peak in the center hole (where a mealworm was placed in training), the monkeys clearly focused their searches along the line that connected the two landmarks. It is possible that the location of the mealworm was more difficult to encode during training when it was not directly adjacent to a landmark(s), as had been the case in Experiment 1 , although the two-landmark task was learned in the same amount of time as the four-landmark task by both monkeys. Biases in the monkeys' searches on the control array tests are somewhat puzzling and may reflect a tendency to use one landmark or the other, rather than the entire configuration, as a cue for the goal. It could be that Elwood adopted a strategy of simply searching on the left side of each landmark. If this were the case, however, a significant difference would be expected between Hole 1 and Hole 4 (the center hole), and this effect was not found. Also, confusion between the two landmarks seems unlikely, given the high contrast between the left and the right walls of the chamber: The right was clear acrylic, and the left was white with a section of wire mesh covered by a wood panel on the outside.

Figure 6 shows the monkeys' search distributions on test trials with expanded arrays in both three-dimensional and two-dimensional formats. The three-dimensional graphs show a tendency for both monkeys to search to the left of the right landmark. ANOVA tests revealed a significant difference in mean searches over the columns of the horizontal dimension for both monkeys [Jake, $F(8,152)=6.16$; Elwood, $F(8,152)=3.42]$. Post hoc Tukey HSD tests conducted on Jake's pattern of searches over columns showed that he searched significantly more in the column immediately to the left of the right landmark (7) than in Columns 1, 8, or 9. Also, Jake searched more in Columns 2, 3, 4, 5, and 6 than in Column 9. Elwood searched significantly more in the column immediately to the left of the right landmark (7) than in Columns 4, 5, or 9. An ANOVA was conducted on the mean searches to just the holes in the center row (2), to further determine where the monkeys focused their searches along this line. Both analyses revealed significant differences across holes for both monkeys [Jake, $F(6,114)=10.35$; Elwood, $F(6,114)=7.06]$. Tukey HSD tests revealed that Jake searched significantly more in the hole directly to the left of the right landmark (7) than in Holes 1, 3, 5, and 9. He also searched significantly more in Hole 6 than in Holes 1, 3, and 9, and more in Hole 5 than in Hole 9. Elwood searched significantly more in the hole directly to the left of the right landmark (7) than in Holes 3, 4, 5, and 9. He also searched more in Holes 1 and 6 than in Hole 9. The results of these analyses illustrate a pattern of searches with a bit more focus on one or two holes along the line that contains the landmarks than was evident with the control array search patterns. Both monkeys seem to be directing searches to the hole just to the left of the right landmark.

As with the control array tests, ANOVA results showed that both monkeys' search patterns differed significantly over rows on the vertical dimension of the expanded array tests [Jake, $F(2,38)=15.97$; Elwood, $F(2,38)=24.23]$. Tukey HSD tests showed that both monkeys searched significantly more in the center row (2) than in Rows 1 or 3. Thus, the high degree of focus on the middle row observed with the control array carried over to expanded array tests as well. The monkeys 


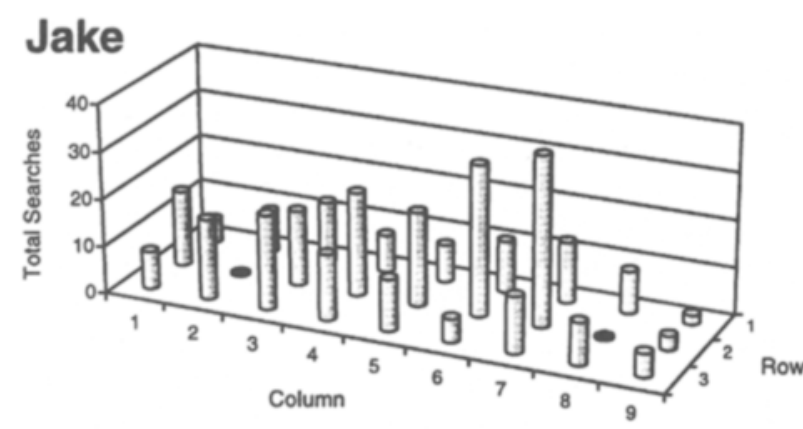

Horizontal
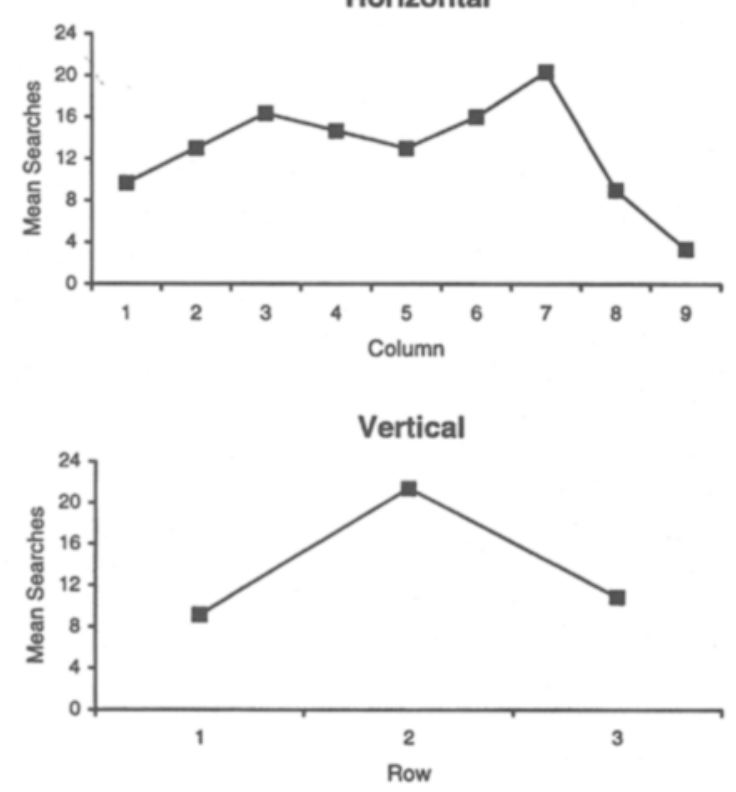

\section{Elwood}

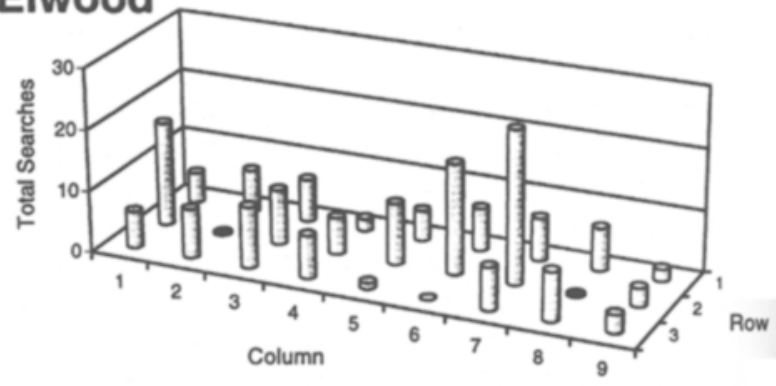

Horizontal
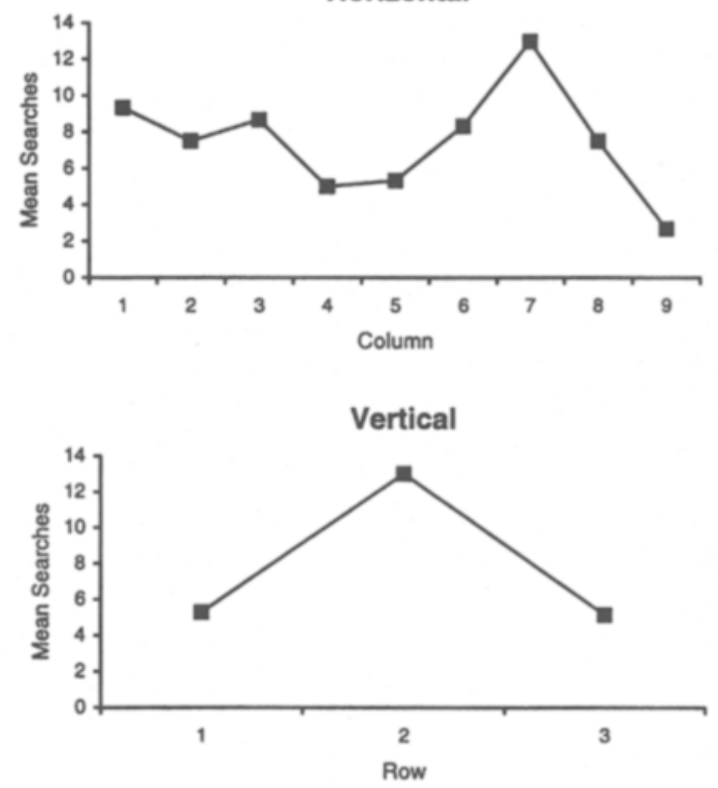

Figure 6. Total searches to each hole within the array boundary and mean searches in columns (horizontal) and rows (vertical) fo Jake and Elwood on test trials with expanded arrays in Experiment 2. Filled black circles denote landmark locations.

seemed to have little problem locating the horizontal line along which the mealworm was found in training.

As for the overall search patterns, it may be that a tendency learned in Experiment 1 to search in a hole adjacent to one or more landmarks carried over to Experiment 2. As in Experiment 1, the monkeys showed a tendency to search more in holes adjacent to landmarks on expanded test trials and did not clearly (or significantly) localize their searching to the center hole. It appears also that the monkeys did not compute the precise direction and distance of the goal from individual landmarks in training, as has been shown with pigeons and gerbils.

\section{EXPERIMENT 3}

Experiments 1 and 2 showed that, although the monkeys directed their foraging to the landmark array, their searches did not seem to be directed to the center hole (the hole baited in training) on expanded test trials. In
Experiment 3, the monkeys were trained with a small and a large-array configuration, and the mealworm wa always buried in the center of each array. It was antici pated that two training arrays might help the monkey develop a conceptual understanding of the spatial rela tionship between the landmarks and the goal, beyon that of simply using the landmarks as a cue for searchin in a general area. That is, the use of two arrays in train ing might aid in the development of a concept of "middle ness" that would carry over to test trials.

The results of studies in which birds were trained wit similar tasks have been mixed. Kamil and Jones (1997 found that food-storing birds (Clark's nutcrackers) contin ued to search in the middle of the landmarks on test trials Spetch, Cheng, and MacDonald (1996) found that pigeon tested with a corresponding procedure on a touch scree did not continue to search in the center of a novel array o test trials. The test data of interest in this experiment wer the monkeys' search patterns on test trials with a landmar array intermediate in size between the two training arrays 


\section{Method}

Subjects and Apparatus. The same squirrel monkeys and apparatus as those used in Experiments 1 and 2 were used in Experiment 3, except that four lengths of wooden doweling covered by green construction paper were used as landmarks.

Procedure. Individual trials proceeded in the same manner as those in Experiments 1 and 2: The monkey entered the test chamber, searched for $1 \mathrm{~min}$, exited the chamber, and received a peanut. The array configurations used in training are depicted in Figure 7. The small array was a configuration of landmarks in a square, with one hole between each landmark. The large array was a square configuration, with five holes between each landmark. A mealworm goal was buried in the center hole of each array. The training procedure was similar to that used in Experiments 1 and 2, except that the two array sizes alternated randomly between trials within a session, with the constraint that the large and small arrays each were presented on three trials (for a total of six trials per session). The configuration was in a different position on the board for each trial.

After about 20 sessions of training, neither monkey was consistently locating the mealworm when it was completely covered by oatmeal. At that point, the training procedure was changed so that a single configuration in a fixed position on the board was presented for 5 trials per daily session. The configuration alternated randomly between the large and the small arrays between sessions. Also, the position of the array on the board varied randomly from session to session. Training proceeded with a partially visible mealworm on the first few trials of Sessions 1-22 for Jake and of Sessions 1-36 for Elwood, at which point the number of trials was increased to 10 per session. Training with one configuration per session continued to Session 37 for Jake and to Session 41 for Elwood (until each monkey found the completely hidden mealworm on every trial for 4-5 sessions in a row). Training then continued with sessions in which the landmark configuration alternated randomly between the small and the large arrays over trials. Five trials were conducted with the small array, and 5 were conducted with the large array, for a total of 10 trials per session. The mealworm was completely buried in the oatmeal on every trial in this final phase of training. Jake continued with this procedure through Session 45, and Elwood continued with it through Session 60 (both monkeys had successfully located the totally buried mealworm on every trial for 5 consecutive sessions by the last session).

Test sessions consisted of nine trials. Six trials were baseline training trials, and three trials were test trials. Of the six baseline trials, three were conducted with the small array, and three were conducted with the large array. Control test trials were conducted with the small array and the large array, as well as with a medium-sized square array with three holes between each landmark; these arrays were not baited with a mealworm. The medium-sized array configuration is depicted in the bottom panel of Figure 7. Each type of array was tested once per session for 20 sessions. The array position on the board varied across trials. The order of the tests and the trials on which the tests were conducted within sessions was determined randomly, with the constraint that the first trial of a session was always a baseline training trial.

\section{Results and Discussion}

In the last five sessions of training, Jake found the completely covered mealworm within 1 min on $92 \%$ of the trials. On small-array trials, he made a mean of 10.40 searches $(S D=6.58)$ before finding the goal, and, on large-array trials, he required a mean of 20.60 searches $(S D=16.01)$ to find the goal. Elwood found the completely covered mealworm within $1 \mathrm{~min}$ on $93 \%$ of the trials in the last five sessions of training. On small-array trials, he made a mean of 4.0 searches $(S D=2.0)$ before

\section{Training Configurations}

Small

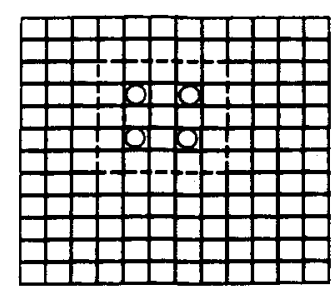

Large

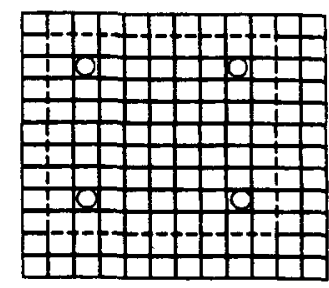

\section{Medium Testing Configuration}

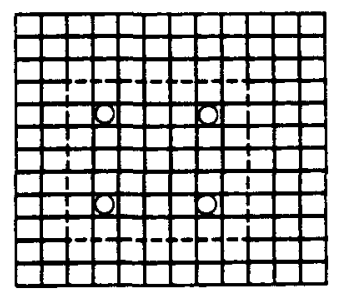

Figure 7. Examples of the large and small configurations used in training (top panel) and of the medium configuration (bottom panel) that was added in testing. Circles show the positions of the landmarks, and the dashed line indicates the landmark boundary within which response patterns were examined.

finding the goal, and, on large-array trials, he needed a mean of 7.0 searches $(S D=2.0)$ to find the goal.

As in the previous experiments, the monkeys' search locations on test trials were analyzed as being either within a one-hole perimeter of the landmark array or beyond this area on the rest of the foraging board, as is illustrated by the dashed lines in Figure 7 . When the small landmark array was used, Jake made a mean of .76 searches per hole $(S D=.25)$ within the landmark boundary and a mean of .05 searches per hole $(S D=.04)$ outside the landmark boundary. On test trials with a large configuration, he made a mean of .35 searches per hole $(S D=$ $.06)$ within the landmark boundary and a mean of .03 searches per hole $(S D=.04)$ on the rest of the board. On the test trials with a medium configuration, he made a mean of .42 searches per hole $(S D=.14)$ within the landmark boundary and a mean of .07 searches per hole $(S D=$ .05 ) outside the landmark boundary. On all three types of test trial, Jake searched more in the holes within the landmark boundary than elsewhere on the board [small configuration, $t(19)=11.93$; large configuration, $t(19)=$ 21.0; medium configuration, $t(19)=10.0$ ]. As in Experiments 1 and 2 , the monkeys did not show any tendency to search the holes within the array boundary in a consistent behavior sequence of visits, and searches outside 
the array boundary followed the same pattern as that in the previous experiments.

On test trials with a small configuration, Elwood made a mean of .38 searches per hole $(S D=.11)$ within the landmark boundary and a mean of .03 searches per hole $(S D=.03)$ outside the landmark boundary. On test trials with a large configuration, he made a mean of .15 searches per hole $(S D=.06)$ within the landmark boundary and a mean of .02 searches per hole $(S D=.02)$ outside the landmark boundary. On test trials with a medium configuration, he made .23 mean searches per hole $(S D=.07)$ within the landmark boundary and .03 mean searches per hole $(S D=.02)$ on the rest of the board. On all three types of test trials, Elwood searched more in the holes within the boundary than elsewhere on the board [small configuration, $t(19)=14.08$; large configuration, $t(19)=10.14$; medium configuration, $t(19)=12.93$ ]

The proportion of searches in the boundary area expected by chance was calculated to be .15 for the small configuration, .55 for the large configuration, and .32 for the medium configuration. Jake directed a higher pro portion of searches to the area within the boundary of test trials with a small configuration $(M=.75, S D=.18)$ on test trials with a large configuration $(M=.95, S D=$ $.06)$, and on test trials with a medium configuration $(M=$ $.76, S D=.17$ ) than would be expected by chance [small $t(19)=15.20$; large, $t(19)=28.61$; medium, $t(19)=$ 11.46]. Elwood also directed a higher proportion o searches to the area within the boundary on test trial: with a small configuration $(M=.69, S D=.17)$, on tes trials with a large configuration $(M=.90, S D=.09)$, anc on test trials with a medium configuration $(M=.82$ $S D=.12$ ) than would be expected by chance [small $t(19)=14.19$; large, $t(19)=18.73$; medium, $t(19)=$ 18.09]. The results of these tests indicate that the mon keys' search patterns were influenced by the position o the landmark array on all three types of test trials.

Figure 8 shows the search distributions within the boundary around the array for both monkeys on contro tests with a small array in three-dimensional and two.
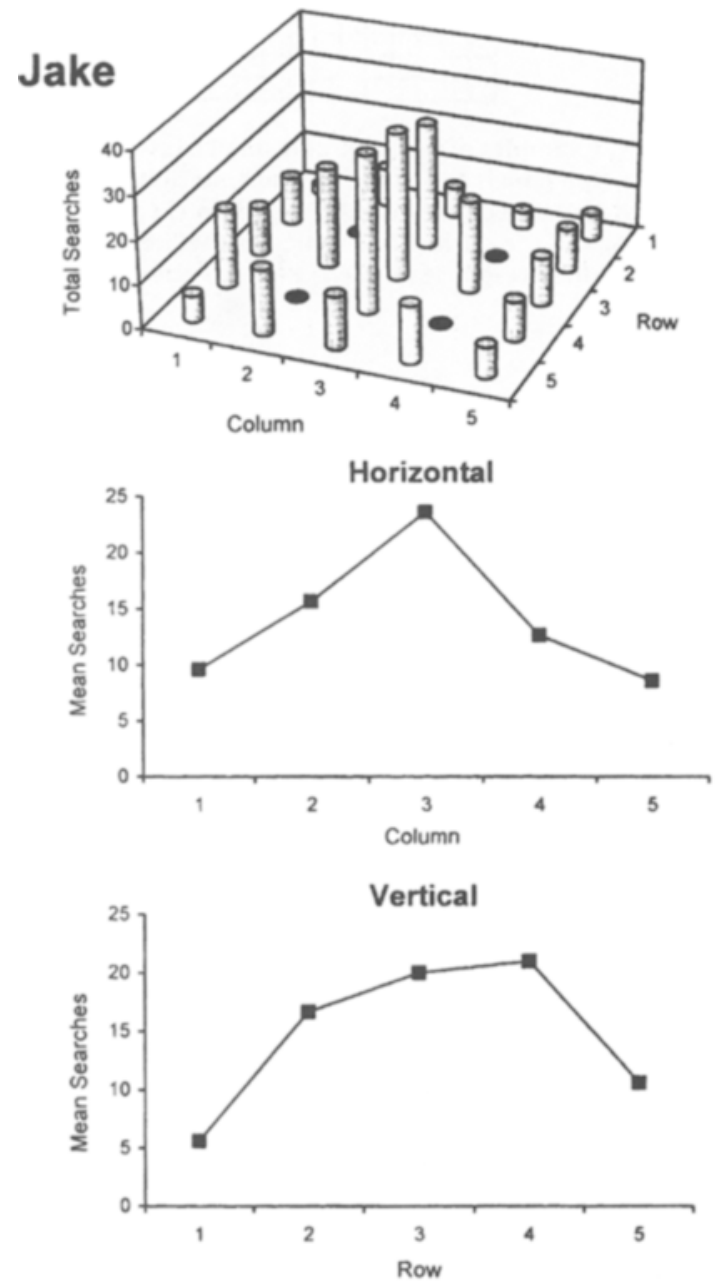
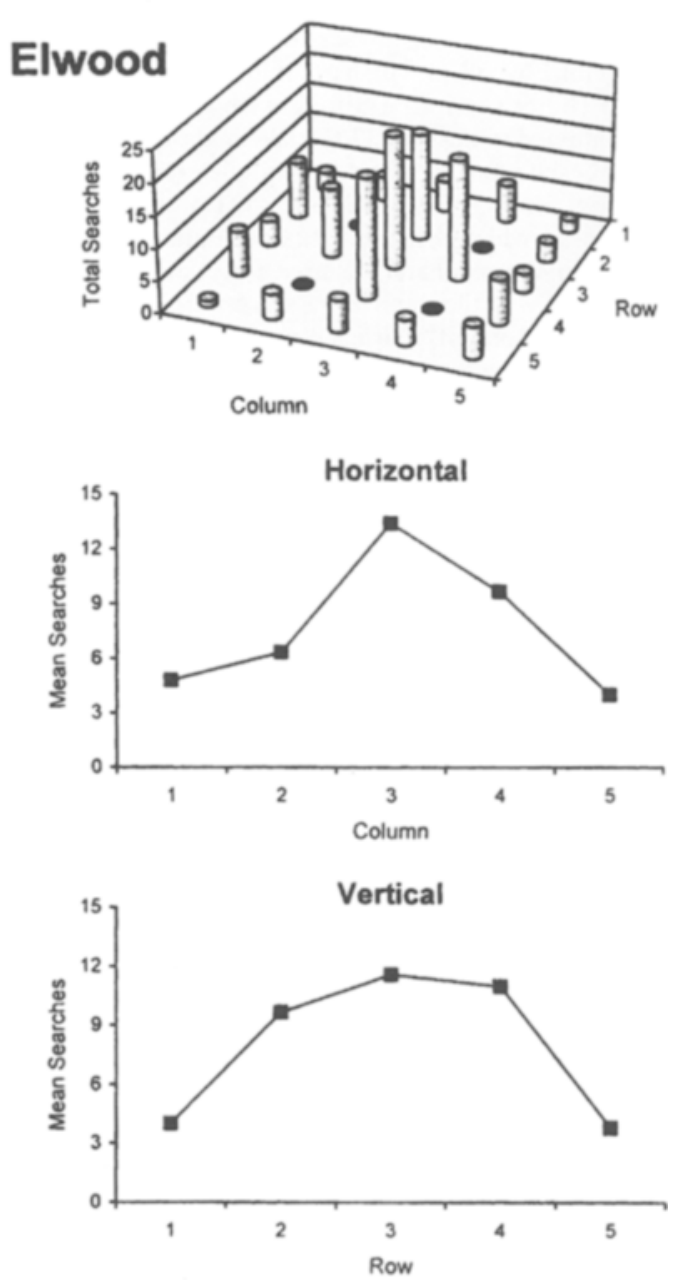

Figure 8. Total searches to each hole within the array boundary and mean searches in columns (horizontal) and rows (vertical) for Jake and Elwood on test trials with a small array in Experiment 3. Filled black circles denote landmark locations. 
dimensional graphs. The three-dimensional graphs show that the monkeys directed a higher total number of searches to the center hole and to the two holes directly above and below it. The two-dimensional patterns of mean searches are similar for both monkeys. An ANOVA conducted on the mean number of searches in each column revealed significant differences across columns for both monkeys [ Jake, $F(4,76)=15.44$; Elwood, $F(4,76)=9.54$ ] . Post hoc Tukey HSD tests revealed that Jake searched significantly more in the center column (3) than in any other column and in Column 2 significantly more than in Column 5. Elwood searched significantly more in the center column than in Columns 1, 2, and 5 and more in Column 4 than in Columns 1 and 5. The general pattern across columns suggests that the monkeys focused on the center column, as they had in tests with the samesized array in Experiment 1.

An ANOVA conducted on the mean searches for rows across the vertical dimension on control tests with a small array also revealed significant differences in the mean number of searches for both monkeys [Jake, $F(4,76)=19.95$; Elwood, $F(4,76)=6.86$ ]. Tukey HSD tests showed that Jake searched more in Rows 3 and 4 than in Rows 1 and 5 and more in Row 2 than in Row 1. Elwood searched significantly more in Rows 2, 3, and 4 than in Rows 1 and 5. Along the vertical dimension, the monkeys' patterns of searching were quite similar and indicated a tendency to search in rows with landmarks and in the center row, but with no significant differentiation among those rows.

Figure 9 depicts the monkeys' search patterns on the control tests with a large array in three-dimensional and two-dimensional layouts. The three-dimensional graphs show a tendency by both monkeys to direct a high number of searches to the right side of the array. This pattern is very clear in the two-dimensional horizontal graphs of the mean searches for each column. ANOVAs carried out on the horizontal dimension searches for both monkeys revealed significant differences across columns [Jake, $F(8,152)=16.39$; Elwood, $F(8,152)=8.22]$. Post hoc

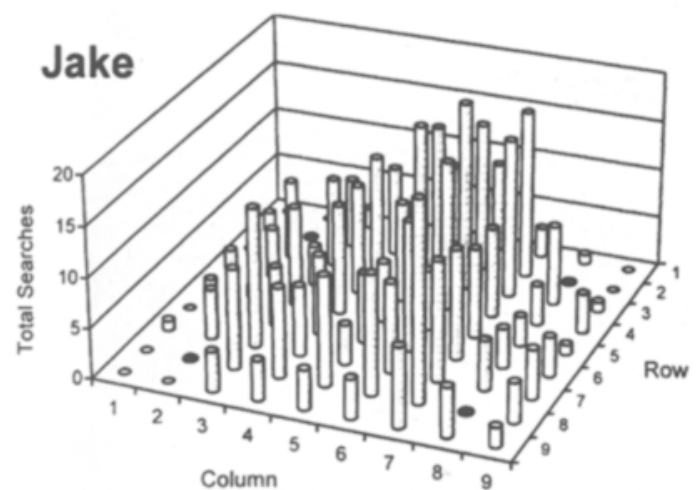

Horizontal
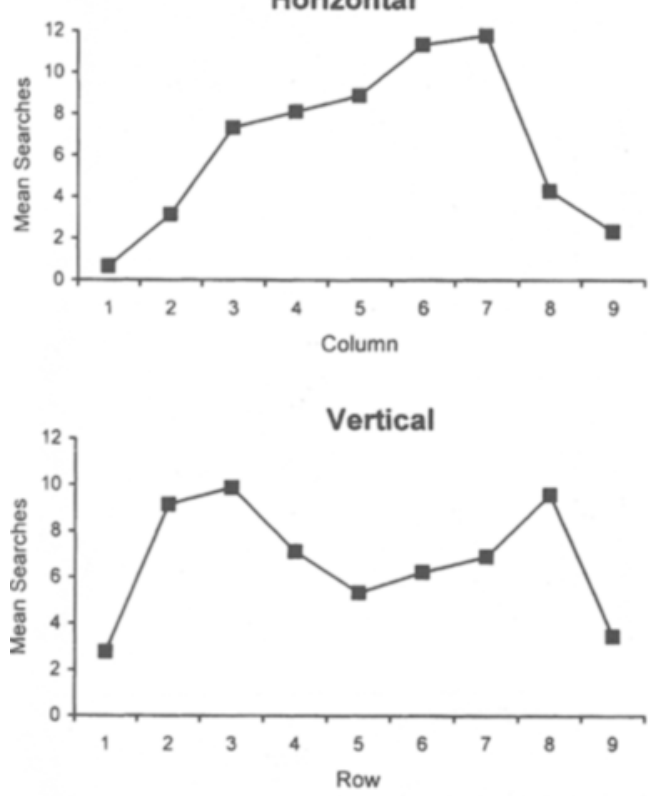
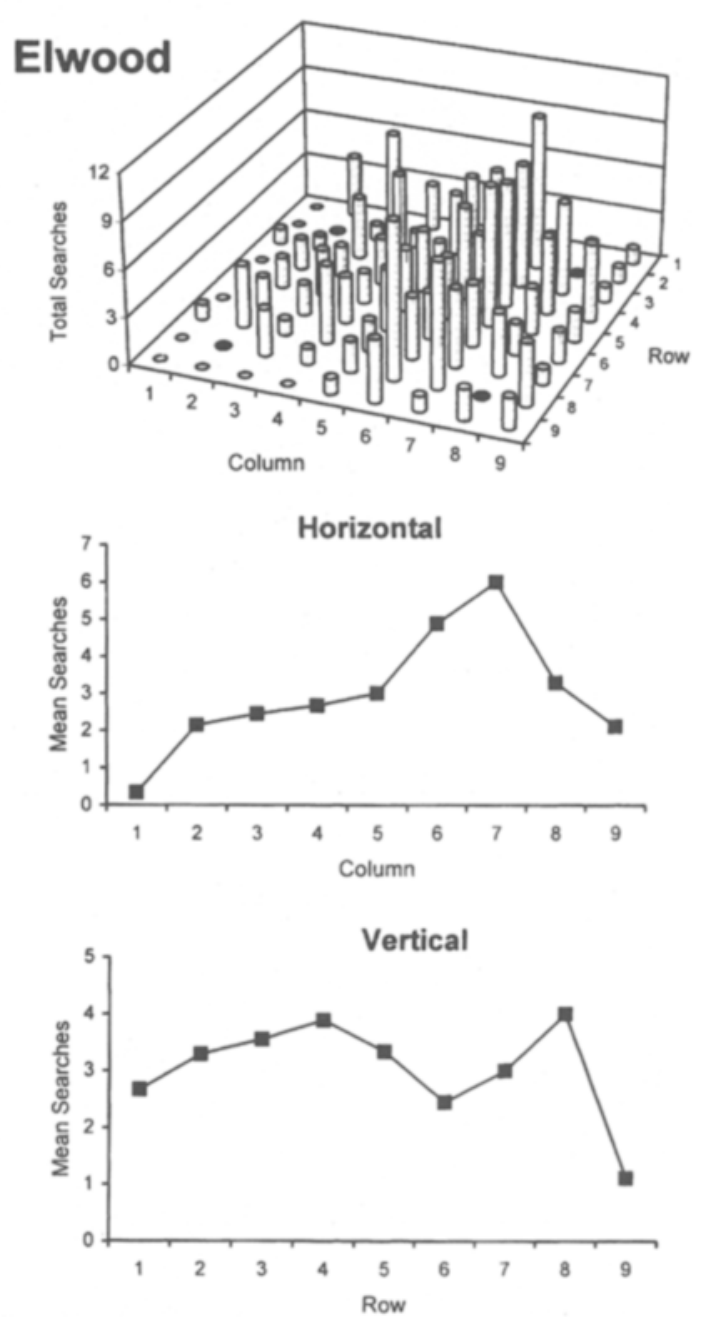

Figure 9. Total searches to each hole within the array boundary and mean searches in columns (horizontal) and rows (vertical) for Jake and Elwood on test trials with a large array in Experiment 3. Filled black circles denote landmark locations. 
Tukey HSD tests showed that Jake searched significantly more in Columns 3, 4, 5, 6, and 7 than in Columns 1, 2, and 9. He also searched more in Columns 5, 6, and 7 than in Column 8 . The majority of his searches along a horizontal dimension seemed to be in the area inside the landmarks (Columns 3-7). Elwood searched significantly more in Column 7 than in Columns $1,2,3,4,5$, and 9 . He also searched more in Columns 5, 6, and 8 than in Column 1 and more in Column 6 than in Columns 2 and 9 . His pattern of searches is more focused on the column with the landmarks on the right and the column directly adjacent to it on the inside.

ANOVAs conducted on the mean number of searches across the vertical dimension for each monkey revealed significant differences across rows for Jake $[F(6,114)=$ 6.54], but not for Elwood. Tukey HSD tests showed that Jake searched significantly more in Rows 2,3 , and 8 than in Rows 1 and 9, indicating that he searched more in the rows with landmarks and in the row directly below the top landmarks than in rows outside the landmarks. There were no significant differences between Rows 2-8, although the pattern of mean searches for these row suggests that more searches were made near the land marks and that there certainly was no tendency to focu on the center row, where the mealworm goal was locate in training. Since the monkeys had been explicitl trained to find a mealworm in the center of the larg array, these results provide some insight into how th monkeys weighted the importance of individual land marks while learning the task. Both appear to have con centrated more on the landmarks on the right side of th array. Perhaps, when failing to find a mealworm on tes trials, they concentrated their searches on the landmark that were most important to their respective strategies

Figure 10 displays three-dimensional and two dimensional search patterns for both monkeys on medium array tests. The three-dimensional depictions of the mon keys' search patterns again do not show a clear peak in : single hole. An ANOVA was carried out on the meal searches in each column across the horizontal dimensiol for each monkey. Both analyses revealed significant dif ferences in the mean searches across columns [Jake
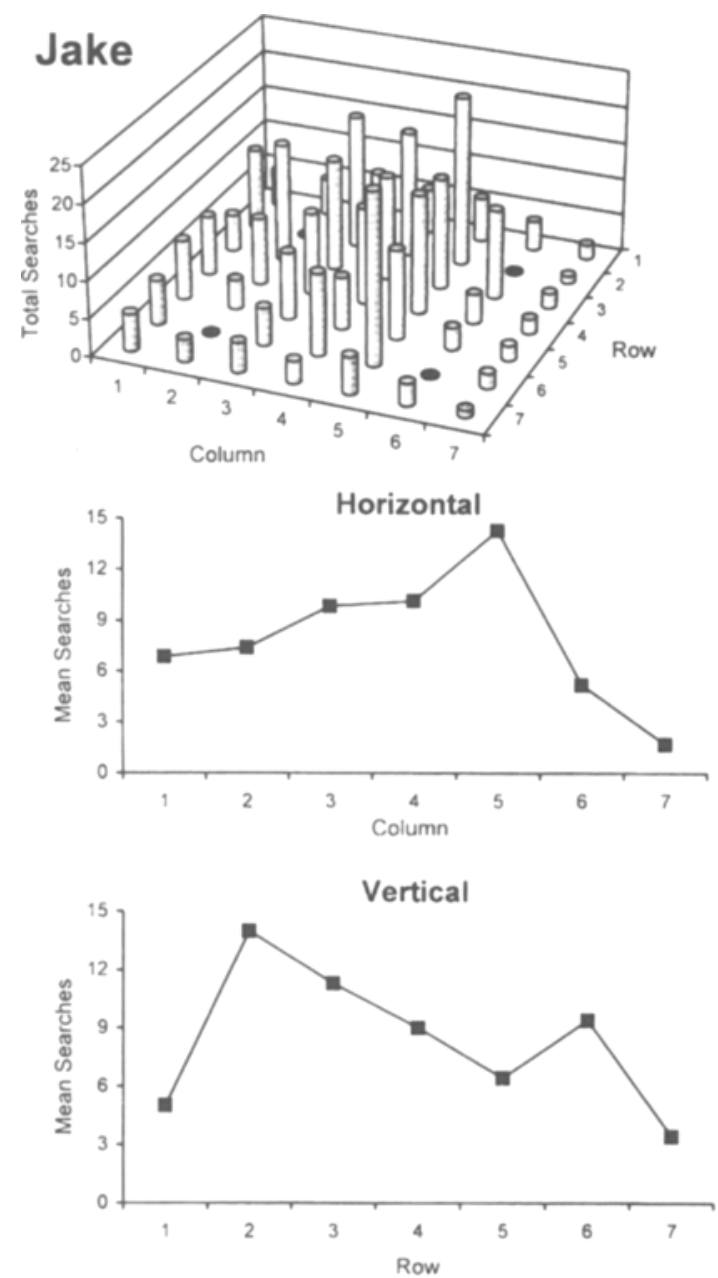
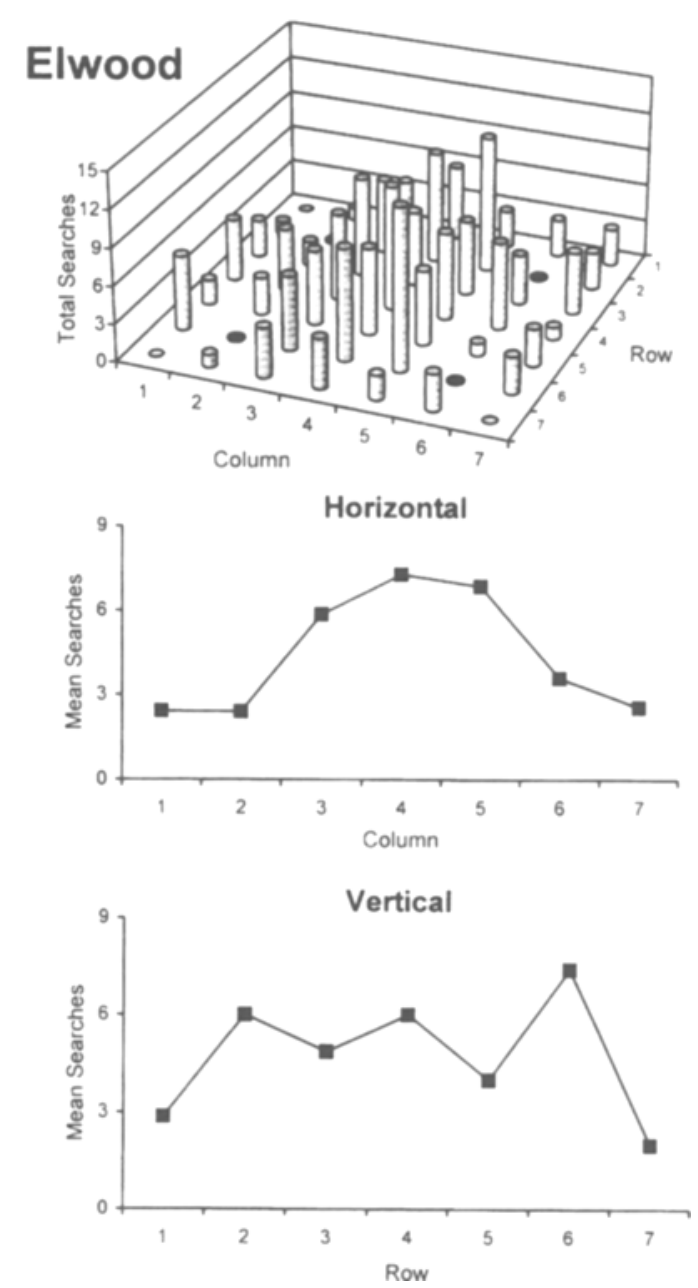

Figure 10. Total searches to each hole within the array boundary and mean searches in columns (horizontal) and rows (vertical) for Jake and Elwood on medium array test trials in Experiment 3. Filled black circles denote landmark locations. 
$F(6,114)=10.14 ;$ Elwood, $F(6,114)=7.04]$. Post hoc Tukey HSD tests showed that Jake tended to search near the landmarks on the right (a similar pattern to that found with the large control array), with significantly more searches being directed to Column 5 than to Columns 1 , $2,3,6$, or 7 . He also searched significantly more in Columns 3 and 4 than in Column 7 . Elwood tended to search in the columns between the landmarks, with significantly more searches in Columns 4 and 5 than in Columns $1,2,6$, and 7 , but no significant differences were found in the mean searches over the central Columns 3-5. On the medium array, he seemed to focus between the set of landmarks along the horizontal dimension, rather than searching more on the right side. There was still no clear peak in the center column, however, so he did not adopt a definite strategy of searching in the center along the horizontal dimension.

An ANOVA was conducted on the mean searches to each row along the vertical dimension for each monkey and revealed significant differences across rows for both monkeys [Jake, $F(6,114)=6.54$; Elwood, $F(6,114)=$ 4.43]. Post hoc Tukey HSD tests revealed that Jake searched significantly more in Rows 2 and 3 than in Rows 1 and 7, in Row 6 significantly more than in Row 7, and in Row 2 significantly more than in Row 5 . He tended to search within the array of landmarks, with the most searches being in Row 2. Elwood's pattern of searches across rows appears unfocused, as is illustrated in Figure 10, but he did search significantly more in Rows 2, 4, and 6 than in Row 7 and significantly more in Row 6 than in Row 1.

Generally, it seems, the larger the array, the more the monkeys exhibited biases to search near a landmark or a set of landmarks on one side (with a few exceptions). Together with the results of Experiment 2, these results suggest that it was very difficult for them to encode the location of the goal relative to an entire configuration of landmarks when the landmarks were not adjacent to the goal in training. As in previous experiments, the monkeys did not localize their searches in the center of the novel test array. Furthermore, the results fail to suggest that they calculated the distance and direction of the goal from individual landmarks in either array.

In summary, even after training with two exemplars (the large and small arrays), the monkeys still seem not to have encoded the location of the goal in terms of "middleness." Instead, the data indicate that both monkeys used the landmarks less precisely to designate a general area within which to search, with the landmark(s) in one area being more salient than others.

\section{GENERAL DISCUSSION}

In Experiments 1 and 2, we investigated the search patterns of two squirrel monkeys with an expanded landmark array configuration after they had learned to locate a hidden mealworm in the center of a smaller configuration. In both cases, they tended to search close to se- lected landmarks instead of in the center (Experiment 1) or made the highest proportion of searches near a selected landmark and fewer searches in the center (Experiment 2). The training procedure in Experiment 3 was set up to help the monkeys develop a more precise representation of the goal location relative to the landmarks by using two different array sizes that maintained the same spatial relationship to the goal. Again, the monkeys did not search more in the center of the novel (mediumsized) array and tended to search near the landmarks instead.

The monkeys' search behavior in Experiments 1 and 2 was different from the behavior of other vertebrate species tested with the same paradigm. Their search patterns did not indicate that they computed the direction and distance of individual landmarks to the goal, as pigeons and gerbils have been observed to do (e.g., Collett et al., 1986; Spetch et al., 1997). They also showed no tendency to search in the center of a landmark configuration that was novel, such as has been demonstrated with humans (Spetch et al., 1997). Overall, however, the monkeys' search patterns more closely resembled those of pigeons and gerbils than those of humans. All three nonhuman species share a tendency to concentrate on the landmarks as separate sources of information, rather than on the entire configuration as a single source of information about the location of the goal. The monkeys differed from pigeons and gerbils in the accuracy with which that information was applied. Their search patterns suggest that they were attracted to the landmarks but used them as beacons and had encoded little about the precise distance and direction relationship between a landmark and the goal.

The effect of training with multiple exemplars of the same landmark/goal spatial relationship in Experiment 3 did not substantially change the monkeys' search behavior on novel configuration test trials. Kamil and Jones (1997) used multiple exemplars to train Clark's nutcrackers to find a seed hidden between two landmarks. The orientation of the landmarks in the room, as well as the distance between them, varied across training trials so that there were 135 correct goal locations across trials in the training phase. The birds were tested with the landmarks at a novel distance apart. The birds both learned to search at the midpoint between the landmarks in training and transferred this ability to novel distances on test trials. Thus, the birds learned the relationship between both landmarks and the goal as a whole configuration. Whether training with such a wide range of exemplars of the same spatial relationship would produce similar search patterns in other birds, especially nonfood-storing species, is not known.

The ability to encode a location at the center of a configuration is, therefore, not limited to humans. Perhaps a procedure similar to that used by Kamil and Jones (1997) with a larger search area might reveal the same ability in squirrel monkeys. The findings of Roberts et al. (1993) raise doubts, however, about the general 
spatial abilities of squirrel monkeys. In those experiments, Jake and Elwood foraged in four laboratory trees placed in a large indoor enclosure. Each tree had 12 holes where raisins could be concealed behind a cover. Specific holes in each tree were consistently baited, and the trees varied in the density of the holes baited. Neither monkey showed any tendency to visit the baited holes or the more densely baited trees first. Subsequently, only one hole on each tree was consistently baited. The number of holes on each tree that the monkeys needed to visit in order to find the raisin improved, but they never consistently went straight to the baited hole. Roberts et al. suggested that the monkeys learned the region of each tree in which a hole was baited but did not learn the exact hole. These results are similar to those of the present study, in that the monkeys' foraging was influenced by the placement of the landmarks in the present experiments but was not precisely focused on one or even a few holes.

A question entertained by Roberts et al. (1993) in consideration of their results also applies to the present findings: Is the foraging strategy of squirrel monkeys masking their spatial abilities in these laboratory tasks? Troops of Saimiri move through the forest and systematically eat all the food found on each tree they encounter. As was suggested by Roberts et al., it may be that the monkeys only need a very general knowledge of the distinctions between geographic regions, and not the ability to pinpoint precise locations. Perhaps, landmarks are used in a very general sense by these monkeys to locate a particular region.

This lack of spatial precision should not be generalized to other nonhuman primates. Studies have shown that chimpanzees (Menzel, 1973), gorillas (MacDonald, 1994), and yellow-nosed monkeys (MacDonald \& Wilkie, 1990) all have an excellent ability to remember the spatial locations of food. It is to be hoped that future research will examine the precise ways in which landmarks are used by apes, Old World monkeys, and New World monkeys. Research of this nature could indicate whether the lack of spatial precision we have found in squirrel monkeys is found in any other species of nonhuman primates and whether it might be related to species that have evolved in a particular ecological niche. A further important questior to be answered is whether any species of nonhuman primate uses multiple landmarks in the same way in which humans have been observed to use them.

\section{REFERENCES}

Baldwin, J. D., \& Baldwin, J. (1972). The ecology and behavior of squirrel monkeys (Saimiri oerstedi) in a natural forest in western Panama. Folia Primatologica, 18, 161-184.

Cartwright, B. A., \& Collett, T. S. (1983). Landmark learning in bees. Journal of Comparative Physiology A, 151, 521-543.

Collett, T. S., Cartwright, B. A., \& Smith, B. A. (1986). Landmark learning and visuo-spatial memories in gerbils. Journal of Comparative Physiology $A, 158,835-851$.

KAMIL, A. C., \& JonEs, J. E. (1997). The seed-storing corvid Clark's nutcracker learns geometric relationships among landmarks. Nature, 390, 276-279.

MACDonald, S. E. (1994). Gorillas' (Gorilla gorilla gorilla) spatial memory in a foraging task. Journal of Comparative Psychology, 108, $107-113$.

MacDonald, S. E., \& WILkiE, D. M. (1990). Yellow-nosed monkeys' (Cercopithecus ascanius whitesidei) spatial memory in a simulated foraging environment. Journal of Comparative Psychology, 104, 382-387.

MenZel, E. W. (1973). Chimpanzee spatial memory organization. Science, 182, 943-945.

Olthof, A., IDEN, C. M., \& Roberts, W. A. (1997). Judgments of ordinality and summation of number symbols by squirrel monkeys (Saimiri sciureus). Journal of Experimental Psychology: Animal Behavior Processes, 25, 325-339.

Phelps, M. T., \& Roberts, W. A. (1994). Memory for pictures of upright and inverted primate faces in humans (Homo sapiens), squirrel monkeys (Saimiri sciureus), and pigeons (Columba livia). Journal of Comparative Psychology, 108, 114-125.

Roberts, W. A. (1998). Principles of animal cognition. Boston: McGraw-Hill.

Roberts, W. A., Mitchell, S., \& Phelps, M. T. (1993). Foraging in laboratory trees: Spatial memory in squirrel monkeys. In T. R. Zentall (Ed.), Animal cognition: A tribute to Donald A. Riley (pp. 131151). Hillsdale, NJ: Errbaum.

Spetch, M. L., Cheng, K., \& MacDonald, S. E. (1996). Learning the configuration of a landmark array: I. Touch-screen studies with pigeons and humans. Journal of Comparative Psychology, 110, 55-68.

Spetch, M. L., Cheng, K., MaCDonald, S. E., Linkenhoker, B. A., Kelley, D. M., \& Doerkson, S. R. (1997). Use of landmark configuration in pigeons and humans: II. Generality across search tasks. Journal of Comparative Psychology, 111, 14-24.

(Manuscript received November 18, 1998; revision accepted for publication July 13,1999.) 